\title{
Risk factors, etiology and prognosis in patients with ischemic stroke and diabetes mellitus
}

Silva-Fernández, J; García-Ruiz, R; García-Ruiz, R; Moreno-Moreno, P; González-Pereira, C. Mancha Centro General Hospital. Alcázar de San Juan (Spain).

Objective: to describe the clinical, etiological and prognostic differences in diabetic and nondiabetic ischemic stroke (IS) patients.

Materials and methods: retrospective analysis of prospective series of ischemic stroke patients. Patients were classified as non-DM and DM patients. Demographic and clinical characteristics were compared between two groups. IS prognosis was evaluated by modified Rankin scale (mRS) and NIHSS (NIH stroke scale).

\section{RESULTS}

\begin{tabular}{|l|c|c|c|}
\hline \multicolumn{1}{|c|}{ Cardiovascular RF } & Non diabetic (71) & Diabetic (53) & $p$ \\
\hline Mean age (years) & $74,00 \pm 11,51$ & $73,95 \pm 14,43$ & 0,98 \\
\hline Men (\%) & 49,2 & 58,4 & 0,18 \\
\hline Hypertensión (\%) & 66,9 & 88,6 & $<0,05$ \\
\hline Hyperlipidemia (\%) & 33,8 & 56,6 & $<0,05$ \\
\hline Ischemic heart disease (\%) & 8,4 & 15 & 0,18 \\
\hline Peripheral arteriopathy (\%) & 2,8 & 3,7 & 0,76 \\
\hline Previous stroke (\%) & 15,4 & 33,9 & $<0,05$ \\
\hline Atrial Fibrilation (\%) & 9,8 & 16,9 & 0,24 \\
\hline Smoke (\%) & 15,4 & 7,5 & 0,13 \\
\hline
\end{tabular}

\begin{tabular}{|c|c|c|c|}
\hline & Non diabetic (71) & Diabetic (53) & $p$ \\
\hline \multicolumn{4}{|l|}{ Stroke subtype } \\
\hline Atherotrombotic (\%) & 14 & 18,8 & \multirow{4}{*}{0,68} \\
\hline Cardiometabolic (\%) & 28,1 & 24,5 & \\
\hline Lacunar (\%) & 18,3 & 20,7 & \\
\hline Indetermined (\%) & 39,4 & 35 & \\
\hline TIA (\%) & 33,8 & 22,6 & 0,7 \\
\hline m Rankin Scale 0-2 (\%) & 76 & 75,4 & 0,94 \\
\hline NIHss $\geq 8$ & 21,1 & 25 & 0,49 \\
\hline
\end{tabular}

\section{Conclusions:}

Our study reveals a higher prevalence of other vascular risk factors in diabetes patients. We have found no differences in relation to IS subtype and IS prognosis. 